\title{
Radiation Resistance Studies of Amorphous Silicon Films*
}

\author{
James R. Woodyard and J. Scott Payson \\ Wayne State University
}

\section{Summary}

Hydrogenated amorphous silicon thin films were irradiated with $2.00 \mathrm{MeV}$ helium ions using fluences ranging from $1 \mathrm{E} 11$ to $1 \mathrm{E} 15 \mathrm{~cm}^{-2}$. The films were characterized using photothermal deflection spectroscopy and photoconductivity measurements. The investigations show that the radiation introduces sub-band-gap states $1.35 \mathrm{eV}$ below the conduction band and the states increase supralinearly with fluence. Photoconductivity measurements suggest the density of states above the Fermi energy is not changing drastically with fluence.

\section{Introduction}

Hydrogenated amorphous silicon alloy (a-Si) thin film photovoltaic devices have been identified for cell module design and development [ref. 1]. The attractiveness of a-Si alloy solar cells for space application is clearly documented and is based on the material's potential for high specific power density, stability and radiation resistance; investigations of a-Si cells show that the radiation resistance is 50 to 100 times more radiation resistant than c-Si, the damage anneals at about 430 $\mathrm{K}$, and that the defect mechanism is probably nuclear knock-on of $\mathrm{Si}$ in the intrinsic layer [ref. 2-5]. The earlier radiation resistance studies of a-Si thin films is reviewed in reference 4 .

Much work remains to be done to develop a basic understanding of the detailed mechanisms involved in radiation resistance, thermal properties, and stability of a-Si based cells. Additionally, the factors limiting cell efficiency must be explored, and a host of engineering and testing activities developed. The work reported herein is concerned with the radiation resistance of a-Si and is a continuation of investigations reported earlier.

This work shifts from the study of devices to thin films because of our need to develop techniques which complement device investigations. Examples of such techniques are photothermal deflection spectroscopy (PDS) and electron spin resonance which are powerful for characterizing thin films, but they are not useful for device structures. We irradiated the films with helium ions instead of protons because of the need to develop techniques with other bombarding ions in order to elucidate the defect generation mechanisms; electronic and nuclear cross section ratio are useful in the identification of defect generation mechanisms. PDS and photoconductivity measurements are carried out to determine the effect of $2.00 \mathrm{MeV}$ helium radiation fluence on the sub-band-gap density of states.

* This work was supported under NASA contract NAG 3-833 and the Wayne State University Institute for Manufacturing Research. 


\section{Experimental}

The a-Si films used in this study were fabricated at Energy Conversion Devices, Inc. using the plasma enhanced chemical vapor deposition method. The films were deposited at a temperature of $543 \mathrm{~K}$ on $25.8 \mathrm{~cm}^{2}$ Corning 7059 glass substrates. The deposition was done at a pressure of 0.25 Torr with a R.F. power of 0.22 Watts $\mathrm{cm}^{-2}$. Pure Silane was used as the feed gas and the films were deposited to a thickness of one micron. Twelve samples were cut to an area of 1.5 by 1.0 $\mathrm{cm}^{2}$, mounted on a sample manipulator, and loaded into a target chamber. The target chamber was evacuated to about $1 \mathrm{E}-7$ Torr. The samples were irradiated with a $2.00 \mathrm{MeV}$ singly ionized helium beam which was 1.0 by $0.6 \mathrm{~cm}^{2}$ in area and had a uniform current density. Ten samples were irradiated with two at each of the following fluences: $1 \mathrm{E} 11,1 \mathrm{E} 12,1 \mathrm{E} 13,1 \mathrm{E} 14$ and $1 \mathrm{E} 15 \mathrm{~cm}^{-2}$. Currents between 20 and 120 nanoamperes were used during the irradiations.

The sub-band-gap optical absorption was measured using PDS [ref. 6,7]. The pump source was a $650 \mathrm{~W}$ tungsten-halogen lamp chopped at $10 \mathrm{~Hz}$, and coupled to the samples through a double monochromator. A thermopile detector was used to monitor the intensity. A laterallysensitive photo-detector was used as a detector; the probe beam was Spectra-Physics model SP-117 laser. The signal was amplified using a Princeton Applied Research model 5301 lock-in amplifier, and the data were collected and averaged using a HP $71 \mathrm{~B}$ computer. The entire optical system was enclosed in a plastic cabinet to reduce noise due to convection currents in the ambient air. The measurements were done at room temperature and the deflecting fluid was carbon tetrachloride. The PDS data were normalized by matching the spectra to absorption coefficients obtained by standard transmission and reflection techniques in the region where the absorption coefficient was between $1 E 3$ and $1 E 4 \mathrm{~cm}^{-1}$.

Figure 1 shows the sub-band-gap absorption coefficient plotted as a function of photon energy for fluences ranging from $1 E 11 \mathrm{~cm}^{-2}$ to $1 \mathrm{E} 15 \mathrm{~cm}^{-2}$. There is no change in absorption between the virgin sample and the sample with a fluence of $1 \mathrm{E} 11 \mathrm{~cm}^{-2}$. The sub-band-gap absorption increases for fluences greater than or equal to $1 \mathrm{E} 12 \mathrm{~cm}^{-2}$. For fluences of $1 \mathrm{E} 14$ and $1 \mathrm{E} 15 \mathrm{~cm}^{-2}$, a pronounced shoulder appears in the sub-band-gap absorption for photon energy of approximately $1.4 \mathrm{eV}$. For lower photon energies, the spectra appear to merge. No change was observed in the above-band-gap absorption as a function of irradiation fluence.

The temperature dependence of the dark conductivity was measured on a virgin sample in the temperature range of 308 to $453 \mathrm{~K}$; it was not possible to measure the irradiated samples as annealing of the defects occurs in this range. The measurements were done in a vacuum of $1 \mathrm{E}-6$ Torr, with an applied bias of $100 \mathrm{v}$. in the co-planar configuration. Silver paint formed the contacts, and the sample was ohmic for applied biases between 0 and $150 \mathrm{v}$. The activation energy for the dark conductivity was found to be $0.75 \mathrm{eV}$.

The room temperature photoconductivity and dark conductivity were measured for all the samples. The measurements were made using white and red illumination sources. An ELH lamp with an infrared absorbing filter was used as the white illumination source; a red color glass filter, which passes wavelengths greater than $600 \mathrm{~nm}$, was used for the red illumination source. Measurements of the photoconductivity as a function of intensity for white and red illumination were performed in air and vacuum in order to separate out any effects due to surface band bending. A vacuum of $1 \mathrm{E}-6$ 
Torr and $2.0 \mathrm{~mm}$ silver paint contacts were employed. A 2.0 by $6.0 \mathrm{~mm}^{2}$ area was scribed in the irradiated area to isolate it from the remainder of the sample area in order to eliminate the effect of the non-irradiated area on the measurements. The contacts were placed within the irradiated area.

The photoconductivity measurements with white and red illumination were essentially the same when corrected for the integrated photon flux. Figure 2 shows the dependence of the photoconductivity on optical density (OD) for red illumination. It is apparent that the photoconductivity decreases as the irradiation increases. Note however, that even though the PDS spectra show no change between the virgin sample and the sample with fluence of $1 \mathrm{E} 11 \mathrm{~cm}^{-2}$, there is a pronounced decrease in the photoconductivity for this fluence. It should also be emphasized that the slope of the photoconductivity versus intensity does not change with fluence, but the photoconductivity decreases monotonically with fluence. The dark conductivity as a function of fluence decreases only slightly with increasing fluence, from approximately $3 \mathrm{E}-10$ to $1 \mathrm{E}-10 \mathrm{~S} / \mathrm{cm}$. For a fluence of $1 \mathrm{E} 15 \mathrm{~cm}^{-2}$ the photoconductivity is, for the largest intensity, only a factor of ten above the dark conductivity.

\section{Discussion}

The optical absorption coefficient is derived from a convolution of the states below the Fermi level and the states above the Fermi-level. In order to derive information about the density of states in the gap, simplifying assumptions must be made about the density of states. In accordance with earlier work [ref. $6,8,9$ ], we have assumed that the matrix element for transitions from a localized state to an extended state is constant and independent of photon energy. For photon energies less than the band gap we assume that only transitions from occupied localized states below the Fermi level to unoccupied extended states in the conduction band are allowed. This implies that the conduction band tail is steeper than the valence band tail, and consequently transitions from extended valence band states to localized conduction band tail states are ignored. Under these conditions the optical absorption is given by [ref. 10]

$$
\alpha=\frac{\text { const }}{\hbar \omega} \times \int N_{v}(E) N_{c}(E+\hbar \omega) d E
$$

where $N_{c}(E)$ corresponds to the density of extended conduction band states, and $N_{v}(E)$ to the occupied localized states below the Fermi-level.

The optical absorption can be modeled with various functions. As shown previously [ref. 12], for high quality amorphous silicon the sub-band-gap absorption is relatively insensitive to the exact form of the deep states and these deep states may be modeled equally well using a peaked mid-gap distribution or an exponential distribution. We chose to model the absorption of the virgin sample with two exponentials. Under these conditions the optical absorption is given by

$$
\alpha=\frac{K}{\hbar \omega} \int_{0}^{E_{F}+\hbar \omega} A\left(E+E^{\prime}\right)^{1 / 2}\left[N_{v} \exp \frac{\left(-E-E_{g}+\hbar \omega\right)}{E_{0 V}}+N_{m} \exp \frac{\left(-E-E_{g}+\hbar \omega\right)}{E_{1}}\right] d E
$$

where $K$ is a constant. $E_{0 v}$ is the slope of the valence-band tail, $E_{1}$ is the slope of the mid-gap distribution, and $E_{\mathbf{g}}$ is the band gap. The form of the conduction band extended states is assumed to be parabolic with the form $A\left(E-E^{\prime}\right)^{1 / 2}$, with $E^{\prime}=0.15 \mathrm{eV}$, as cited in references 11 and 12 . 
The effect of irradiation on the absorption is modelled by adding to equation 2 a peak in the density of states in the form used by Wronski et al. [ref. 8]. The excess absorption due to the irradiation is given by

$$
\alpha^{\prime}=\frac{K}{\hbar \omega} \int_{0}^{E_{\mathrm{r}}+\hbar \omega} A\left(E-E^{\prime}\right)^{1 / 2} N_{\mathrm{r}} \operatorname{sech}\left[\frac{\left(-E-E_{\mathrm{r}}+\hbar \omega\right)}{E_{\mathrm{r} 0}}\right] d E
$$

where $\mathrm{N}_{\mathrm{r}}$ is the peak value of the distribution, $\mathrm{E}_{\mathrm{r}}$ is the position of the peak with respect to the conduction band, and $\mathrm{E}_{\mathrm{r} 0}$ is the width of the distribution. $\mathrm{K}, \mathrm{A}$ and $\mathrm{E}_{0}$ are the same as was defined earlier. This distribution was chosen in order to fit the absorption data by changing only the peak value of the distribution and not varying the width. The total absorption is then given by

$$
\alpha_{\mathbf{T}}=\alpha+\alpha^{\prime}
$$

It was found that if we model the density of states with a single exponential and a peaked mid-gap distribution that the width of the mid-gap distribution changed by more than a factor of two in going from the virgin sample to the most highly irradiated sample. It is found that a good fit to the data is achieved placing the peak in the density of states at $1.35 \mathrm{eV}$ below the conduction band. The position of this peak in the density of states agrees well with the position of the defect peak reported by Jackson et al.[ref. 13], but disagrees with the work of Vanáček et al. [ref. 9] who places the defect peak at $1.0 \mathrm{eV}$ below the conduction band. Since there was no change in the optical absorption for the sample irradiated with $1 \mathrm{E} 11 \mathrm{~cm}^{-2}$, we cannot derive any information about the increase in the density of states from absorption measurements.

The increase in the density of states due to the irradiation, $\mathbf{n r}$, is easily calculated from the integration of the $\operatorname{sech}(x)$ in equation 2 . This is plotted versus fluence in figure 3 . For fluences larger than $1 \mathrm{E} 13 \mathrm{~cm}^{-2}$ the defect density follows a power law with exponent equal to $\sim 1.5$. The optical absorption data were examined within the model proposed by Jackson et al. [ref. 6,13]. The defect density in this model is calculated by subtracting the Urbach edge absorption from the total absorption and integrating this excess absorption, $\alpha_{\text {ex }}$. The number of defects is given by

$$
\mathrm{N}_{\mathrm{s}}=7.9 \times 10^{15} \int \alpha_{\mathrm{ex}}(\mathrm{E}) \mathrm{dE}
$$

This is also plotted in figure 3 . Note that the number of defects does not obey the same functional form, and seems to be saturating at higher fluences. At low fluence the two models give the same results.

An indication of the disorder in the amorphous silicon alloy is seen in the slope of the Urbach edge [ref. 14]. We find that the slope of the Urbach edge increases only slightly from $E_{0}=0.055 \mathrm{eV}$ for low fluence to $E_{0}=0.070 \mathrm{eV}$ for a fluence of $1 \mathrm{E} 15 \mathrm{~cm}^{-2}$, as shown in figure 4 . If these same data are examined within the model of Jackson et al. [ref. 6,13], the slope increases dramatically with fluence, reaching a value of $E_{0}=0.117 \mathrm{eV}$ for the maximum fluence. This would seem to indicate a substantial change in the structure of the material with fluence, whereas the slope as calculated in our model would indicate only slight changes. 
In summary, our model indicates that the result of irradiation is to increase the density of states near $E_{c}-E=1.35 \mathrm{eV}$, and even for the highest fluence, indicates only a slight increase in the disorder of the system as revealed by the slope of the Urbach edge. The increase in the defect density follows a power law with exponent of $\sim 1.5$. A comparison with Jackson's model [ref. 1,8], would indicate that the irradiation also increases the density of defects, but not in the same way, and that the disorder in the system, as modeled by the slope of the Urbach edge of the absorption coefficient, indicates a large change in the disorder with increasing fluence, as compared to a relatively small change for our model.

One may also obtain information about the gap-states in a-Si:H through analysis of the steadystate photoconductivity. Unlike the sub-band-gap optical absorption, the steady-state photoconductivity probes the region in the band-gap from the Fermi level towards the conduction band. It has been shown that if the trap distribution is exponential between the Fermi level and the trap quasi-Fermi level [ref. 15], the intensity dependence of the photoconductivity obeys the relation $\sigma_{\mathrm{ph}} \propto \mathrm{I}^{\gamma}$, where $\gamma$ is determined by the logarithmic slope of the distribution of states. Referring to figure 2 we note that the value of $\gamma$ for the virgin sample is 0.82 . A fluence of $1 \mathrm{E} 11 \mathrm{~cm}^{-2}$ results in $\gamma$ increasing to 0.87 . Increasing the fluence results in very little change in the logarithmic slope of the photoconductivity versus intensity curves. This indicates that the form of the trap distribution is not changing drastically with fluence. The values of the photoconductivity are, however, seen to decrease with fluence. Using the generalized Shockley-Read-Hall analysis of Taylor and Simmons [ref. 16], we note that the recombination rate for an arbitrary distribution of states in the band gap is proportional to the number of states that lie between the trap quasi-Fermi level for holes and the trap quasi-Fermi level for electrons, and for an n-type material most of the recombination takes place between the Fermi level and the trap quasi-Fermi level for holes. If we associate the increase in the optical absorption due to irradiation with an increase in the density of states below the Fermi level, then one would expect the photoconductivity to decrease dramatically for fluences of $1 \mathrm{E} 13 \mathrm{~cm}^{-2}$ and above, which is indeed observed as shown in figure 4 . However, note that even for a fluence of $1 \mathrm{E} 11 \mathrm{~cm}^{-2}$, there is a decrease in the photoconductivity of almost a factor of 10 . This might be due to an increase in the density of states above the Fermi level, which is not probed by the sub-band-gap absorption. One possible method to confirm this conjecture would be to fabricate $\mathrm{n}+\mathrm{-i}-\mathrm{n}+$ devices and measure the space-charge-limited conduction as a function of fluence, which gives information about states in the gap from the Fermi level towards the conduction band [ref. $17,18]$. We should like to note that the decrease in the photoconductivity with increasing fluence can not be due to the de-sensitization of the photoconductivity [ref. 19] when the Fermi level falls towards mid-gap, because the dark conductivity changed by less than a factor of ten with even the highest fluence. We therefore conclude that the increase in the sub-band-gap absorption is due to an increase in the density of states below the Fermi level and these states act as recombination centers causing an increase in recombination and thus a decrease in the photoconductivity.

Preliminary annealing measurements indicate that the thin film defects anneal at about the same temperature as devices. This fact along with the measurements reported herein suggests that studies of thin films are useful in the elucidation of the mechanisms which lead to the degradation of devices subjected to irradiation.

\section{Conclusion}

Our model indicates that irradiation increases the density of states near $\mathrm{Ec}-\mathrm{E}=1.35 \mathrm{eV}$ and there is only a slight increase in the disorder of the system as revealed by the slope of the Urbach 
edge. The increase in the sub-band-gap absorption is due to an increase in the density of states below the Fermi level and these states act as recombination centers causing an increase in recombination and thus a decrease in the photoconductivity. Thin film studies appear to be important for learning the basic aspects of device degradation.

\section{References}

[1] Joseph Wise and Cosmo Baraona, Proc. of the Space Photovoltaic Research and Technology Conference, Nasa Conference Publication 2475, 1986, page 355.

[ 2] Joseph J. Hanak, Art Myatt, Prem Math and James R. Woodyard, Proc. of the Eighteenth IEEE Photovoltaic Specialists Conference, 1985, page 1718.

[ 3] J. J. Hanak, Englade Chen, C. Fulton, A. Myatt and J. R. Woodyard, Proc. of the Space Photovoltaic Research and Technology Conference, NASA Conference Publication 2475, 1986, page 99.

[ 4] J. J. Hanak, E. Chen, Art Myatt and James R. Woodyard, Proc. of the Nineteenth IEEE Photovoltaic Specialists Conference, 1987, page 630.

[ 5] James R. Woodyard and J.J. Hanak, "Amorphous Silicon Semiconductors-Pure and Hydrogenated", Materials Research Society Symposia Proceedings, Edited by D. Adler, A. Madan, Y. Hamakawa and M. Thompson, 1987, Volume 95, page 533.

[6] N.M. Amer and W. B. Jackson, Appl. Phys. A. 32, 141 (1983).

[ 7] J. S. Payson and S. Guha, Phys. Rev. B 32, 1326 (1985).

[ 8] C. R. Wronski, B Abeles, T.Tiedje and G. B. Cody, Solid State Commun. 441423 (1982).

[ 9] M. Vanecek, J. Kocka, J. Stuchlik, Z. Kozisek, O. Strika, and A. Triska, Sol. Energy Mater. 8 411 (1983).

[ 10] N. F. Mott and E. A. Davis, Electronic Processes in Non-Crystalline Solids (Clarendon, Oxford, 1979).

[ 11] C.-Y. Huang, S. Guha, and S. J. Hudgens, Phys. Rev. B 27, 7460 (1983).

[ 12] W. E. Spear, D. Allan, P. G. LeComber and A. Ghaith, J. Non-Cryst. Solids 35-36, 357 (1980).

[ 13] W. B. Jackson and N. M. Amer, Phys. Rev. B 25, 5559 (1982).

[ 14] G. D. Cody, T. Tiedje, B. Abeles, B. Brooks and Y. Goldstein, Phys. Rev. Lett. 47, 1480 (1981).

[15] A. Rose, Concepts in Photoconductivity and Allied Problems (Krieger, New York, 1978).

[ 16] J. G. Simmons and G.W. Taylor, Phys. Rev. B 4, 502 (1971).

[ 17] W. den Boer, J. Physique 42, C4, 451 (1981).

[ 18] K. D. Mackenzie, P. G. LeComber, and W. E. Spear, Phil. Mag. B 46, 377 (1982).

[ 19] M. Hack, S. Guha, and M. Shur, Appl. Phys. Lett. 45, 467 (1984). 


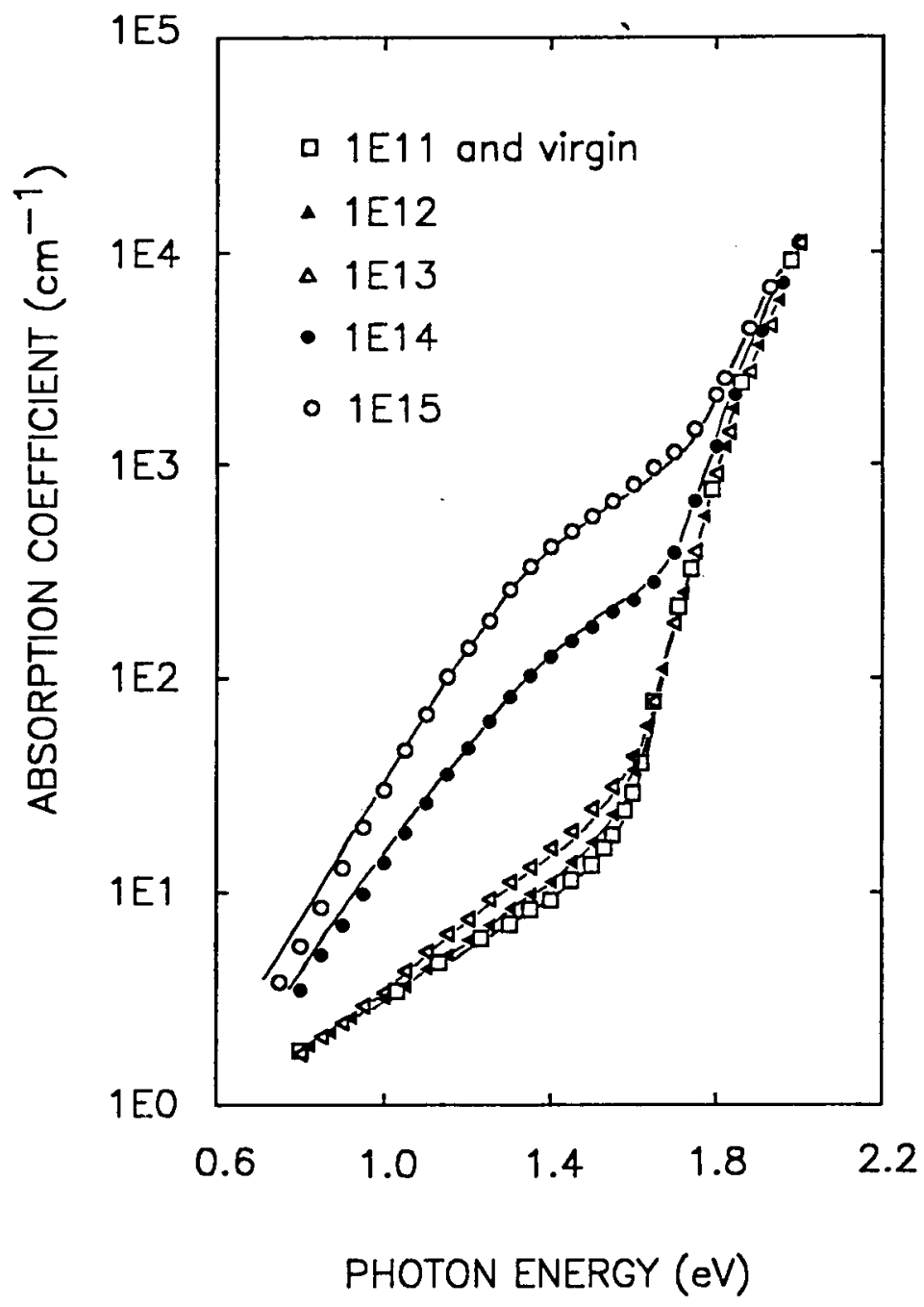

Figure 1. The optical absorption coefficient as a function of incident photon energy for various fluence, where the open circles correspond to a fluence of--1E15 cm-2, closed circles--1E14 cm-2, open triangles-$1 E 13 \mathrm{~cm}-2$, closed triangles--1E12 cm-2, and the open squares--1E11 $\mathrm{cm}-2$ and the virgin sample. The solid lines are absorption coefficients calculated from the density of states model. 


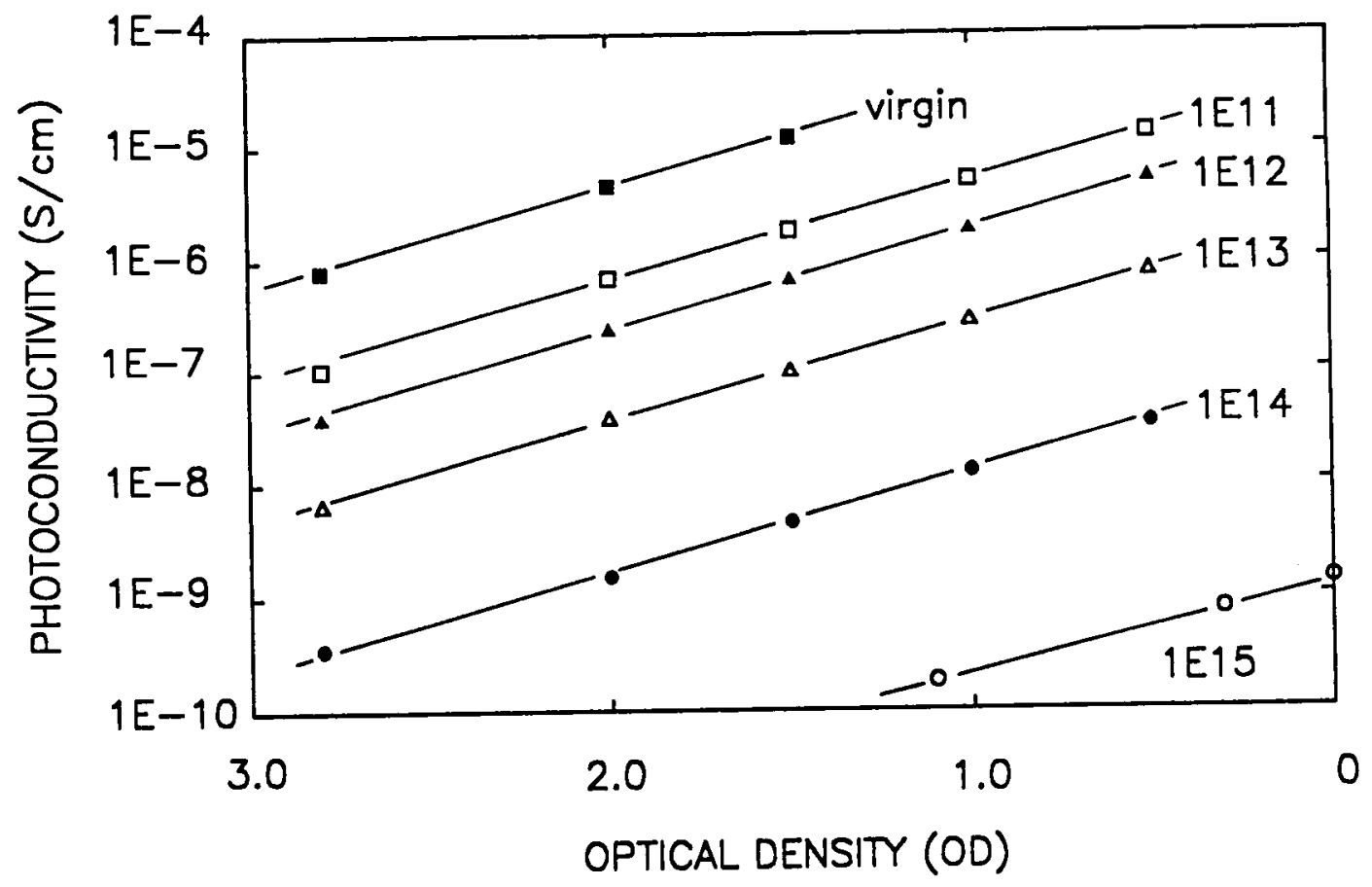
Figure 2. The steady-state photoconductivity as a function of $\mathrm{cm}^{-1}$, closed circles--1E14 $\mathrm{cm}-2$, the open triangles--1E13 cm-2, the closed triangles--1E12 cm-2, the open squares--1E1I cm-2, and the closed squares corresponding to the virgin sample. 


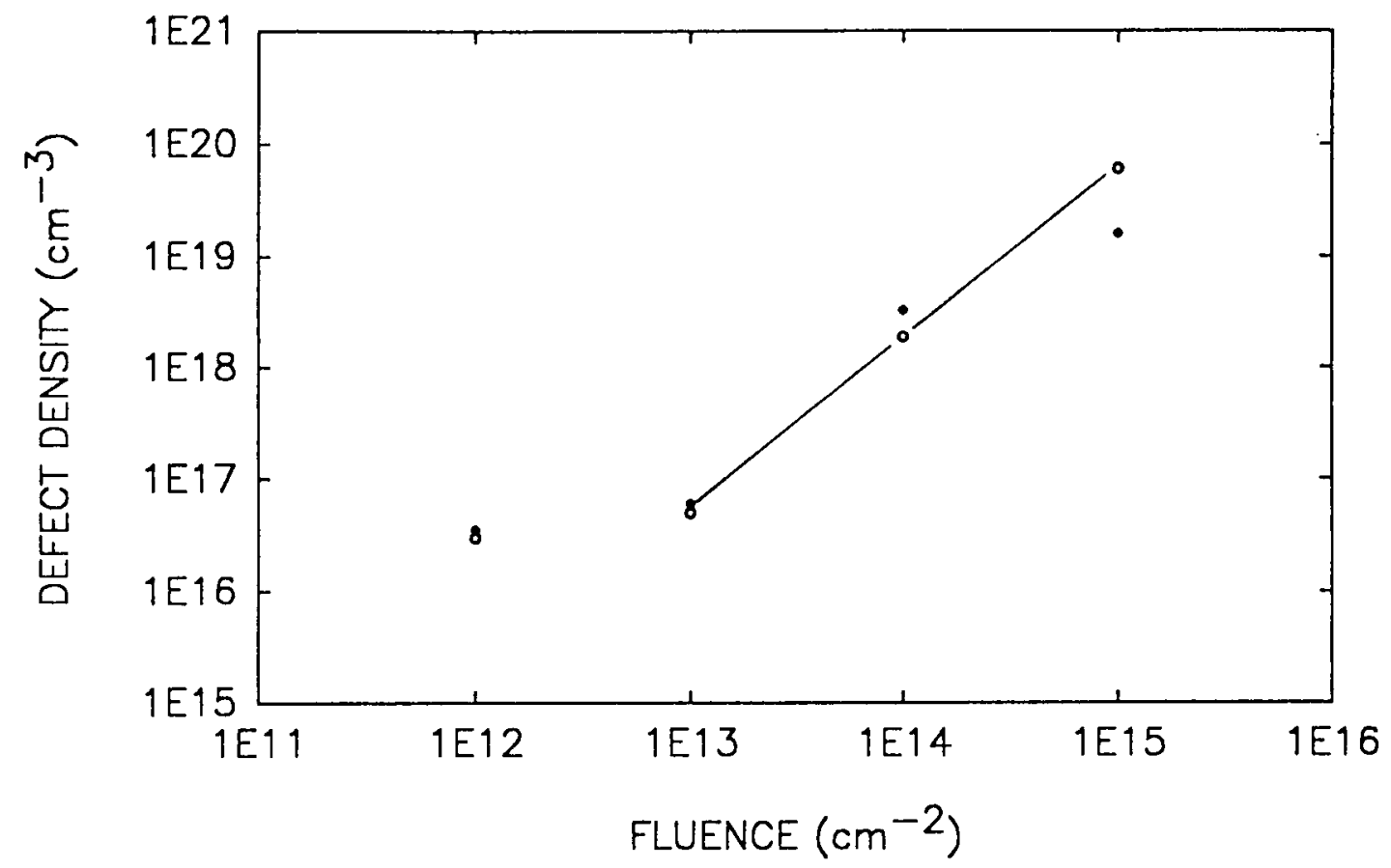

Figure 3. The ion-induced defects versus ion fluence. The open circles were calculated using our model, whereas the closed circles were calculated using the model of Jackson et al. (ref. 6,13).

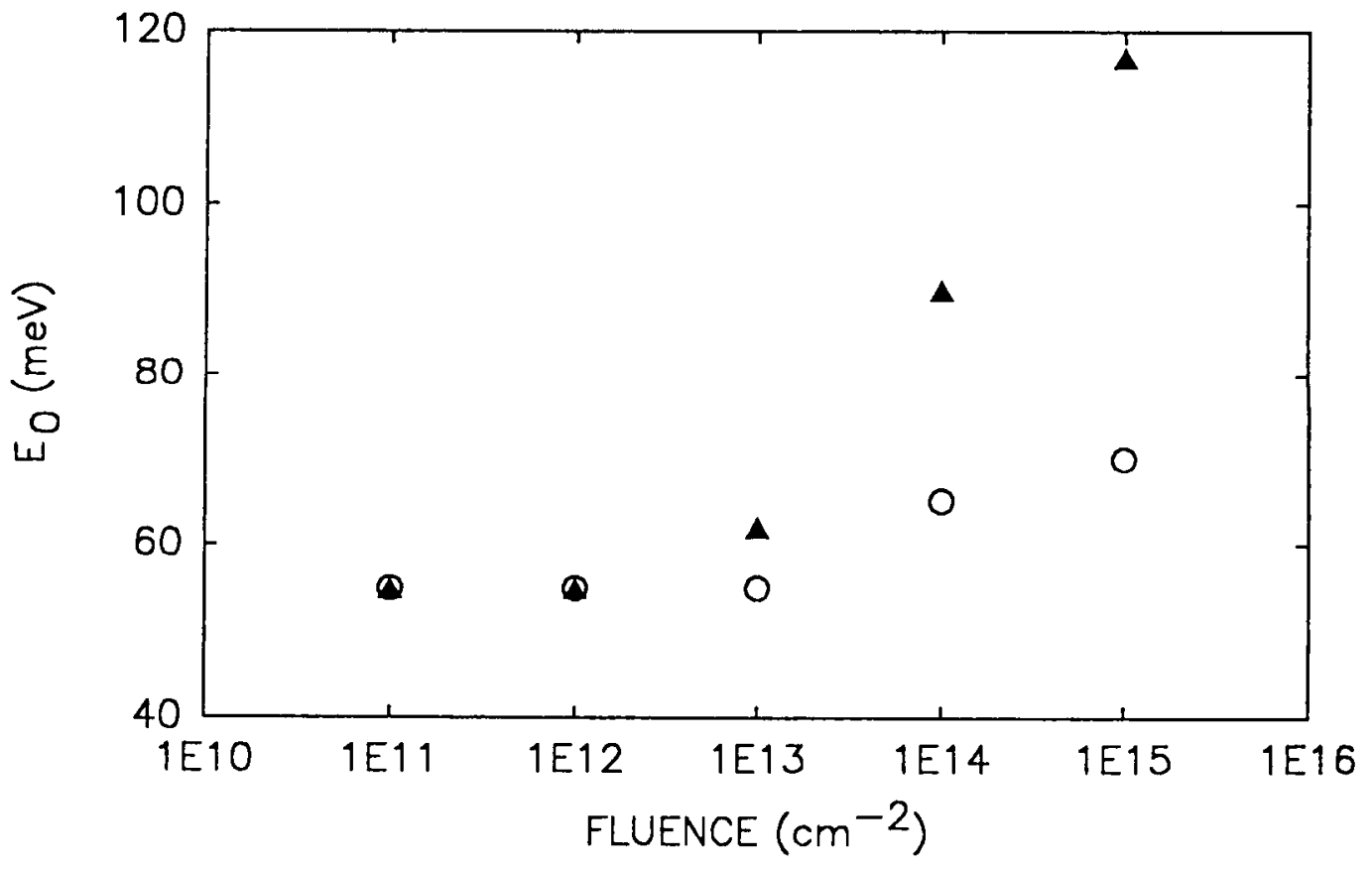

Figure 4. The logarithmic slope of the Urbach edge of the density of states as calculated using our model (open circles) and the model of Jackson et al. (ref. 6,13), (diamonds). 